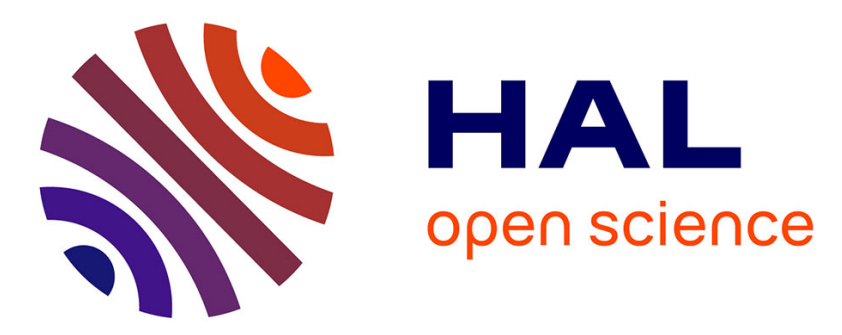

\title{
Sonic hedgehog mediates a novel pathway of PDGF-BB-dependent vessel maturation
}

\author{
Qinyu Yao, Marie-Ange Renault, Candice Chapouly, Soizic Vandierdonck, \\ Isabelle Belloc, Beatrice Jaspard-Vinassa, Jean-Marie Daniel-Lamazière, \\ Muriel Laffargue, Aksam Merched, Claude Desgranges, et al.
}

\section{To cite this version:}

Qinyu Yao, Marie-Ange Renault, Candice Chapouly, Soizic Vandierdonck, Isabelle Belloc, et al.. Sonic hedgehog mediates a novel pathway of PDGF-BB-dependent vessel maturation. Blood, 2014, pp.123(15)2429-2437. inserm-01159066

\section{HAL Id: inserm-01159066 https://www.hal.inserm.fr/inserm-01159066}

Submitted on 2 Jun 2015

HAL is a multi-disciplinary open access archive for the deposit and dissemination of scientific research documents, whether they are published or not. The documents may come from teaching and research institutions in France or abroad, or from public or private research centers.
L'archive ouverte pluridisciplinaire $\mathbf{H A L}$, est destinée au dépôt et à la diffusion de documents scientifiques de niveau recherche, publiés ou non, émanant des établissements d'enseignement et de recherche français ou étrangers, des laboratoires publics ou privés. 


\section{Sonic hedgehog mediates a novel pathway of PDGF-BB- dependent vessel maturation}

Yao Qinyu ${ }^{1,2}$, Renault Marie-Ange ${ }^{1}$, Chapouly Candice ${ }^{1,2,3}$, Vandierdonck Soizic ${ }^{1,2,3}$, Belloc Isabelle $^{1}$, Jaspard-Vinassa Beatrice ${ }^{1,2}$, Daniel-Lamazière Jean-Marie ${ }^{1}$, Laffargue Muriel ${ }^{5}$, Merched Aksam ${ }^{1,4}$, Desgranges Claude ${ }^{1}$ and Gadeau Alain-Pierre ${ }^{1}$

1) INSERM, Cardiovascular adaptation to ischemia, U1034, F-33600 Pessac, France;

2) Université de Bordeaux, Cardiovascular adaptation to ischemia, UMR1034, F-33600 Pessac, France;

3) Department of Clinical Pharmacokinetics, Haut-Lévêque Hospital, CHU Bordeaux, Pessac, F33604, France;

4) Department of Molecular and Cellular Biology, Baylor College of Medicine, 77030 Houston TX, USA;

5) INSERM, U1048-I2MC, Université de Toulouse, F-31432 Toulouse, France. 


\begin{abstract}
$\underline{\text { Abstract }}$
Recruitment of mural cells, i.e. pericytes and smooth muscle cells (SMCs), is essential to improve the maturation of newly formed vessels. Sonic hedgehog ( $\mathrm{Shh}$ ) has been suggested to promote the formation of larger and more muscularized vessels, but the underlying mechanisms of this process have not yet been elucidated. We first identified Shh as a target of PDGF-BB and found that SMCs respond to Shh by upregulating ERK1/2 and Akt phosphorylation. We next showed that PDGF-BB-induced SMC migration was reduced after inhibition of Shh or its signaling pathway. Moreover, we found that PDGF-BB-induced SMC migration, involves Shh-mediated motility. In vivo, in the mouse model of corneal angiogenesis, Shh is expressed by mural cells of newly formed blood vessels. PDGF-BB inhibition reduced Shh expression, demonstrating that Shh is a target of PDGF-BB, confirming in vitro experiments. Finally, we found that in vivo inhibition of either PDGF-BB or Shh signaling reduces $\mathrm{NG}^{+}$mural cell recruitment into neovessels and subsequently reduces neo-vessel lifespan. Our findings demonstrate, for the first time, that Shh is involved in PDGF-BB-induced SMC migration and recruitment of mural cells into neo-vessels and elucidate the molecular signaling pathway involved in this process.
\end{abstract}




\title{
Key points
}

1) Maturation of VEGF-induced new vessels in cornea involves a PDGF-Sonic Hedgehog axis

2) Sonic hedgehog promotes PDGF-BB-mediated smooth muscle cell migration by inducing Erk1/2 and PI3K $\gamma$ activation and an increased motility

\author{
Abbreviations \\ Dhh: Desert hedgehog: \\ EC: Endothelial Cells \\ FGF-2: Fibroblast Growth Factor-2 \\ GPCR: G protein-coupled receptors \\ HB-EGF: Heparin-Binding EGF-like Growth Factor \\ Ihh: Indian hedgehog \\ MC: Mural Cells \\ MCP-1: Monocyte Chemotactic Protein-1 \\ Gli: Glioma-associated oncogene \\ PDGF-BB: Platelet-Derived Growth Factor-BB \\ PDGFR- $\beta$ : PDGF receptor $\beta$ \\ Ptch-1: Patched-1 \\ SMC: Smooth Muscle Cell \\ Smo: Smoothened \\ Shh: Sonic hedgehog \\ VEGF: Vascular Endothelial Growth Factor
}




\section{$\underline{\text { Introduction }}$}

Formation of new vessels occurs during early development and adulthood, and also in pathological conditions such as ischemia, tissue regeneration, inflammation and cancer ${ }^{1}$. Mural cell (MC) recruitment is essential for the stability and the functionality of newly formed vessels, particularly, by controlling endothelial cell (EC) survival through the regulation of Ang-1/Ang-2 balance. MCs include pericytes and smooth muscle cells (SMCs) although a clear distinction between these two cell types remains controversial ${ }^{2}$. Mechanisms involved in their recruitment on endothelial sprouts are complex. Pericytes are proposed to originate from surrounding mesenchymal precursors or to be recruited from the wall of adjacent vessels before differentiating into SMCs ${ }^{3}$. It is also postulated that proliferation and migration of MCs towards ECs is dependent on a precisely orchestrated gradient of soluble chemotactic factors generated from ECs in their microenvironment.

Platelet-Derived Growth Factor-BB (PDGF-BB) is known to promote MC recruitment ${ }^{4-7}$. It is secreted by ECs, upon angiogenic stimulation ${ }^{8,9}$ or hypoxia ${ }^{10}$. It is a potent chemotactic and growth factor for both vascular SMCs and pericytes expressing PDGF receptor $\beta$ (PDGFR- $\beta)^{5}$. Hedgehog (Hh) proteins, namely Sonic, Indian and Desert Hh (Shh, Ihh and Dhh respectively), are involved in vessel formation during development ${ }^{11}$ and ischemia-induced post-natal neovascularization ${ }^{12,13}$. Interestingly, in addition to increasing capillary density, Shh gene therapy in the ischemic myocardium was shown to promote the formation of larger and more muscularized blood vessels ${ }^{14}$. Moreover, Shh was shown to upregulate MC-related markers during the formation of microvessel-like structures in vitro ${ }^{15}$ suggesting a potential action of Shh in vessel maturation. At the cellular level, recombinant Shh was shown to induce SMC and pericyte proliferation and survival ${ }^{16-19}$ and to contribute to PDGF-BB-induced myofibroblast proliferation ${ }^{20}$. Even though Shh was shown to induce the migration of various cell types such as fibroblasts ${ }^{21}$, ECs ${ }^{22}$, endothelial progenitors ${ }^{23}$, and monocytes ${ }^{24}$, its role and mechanism in SMC migration in vitro and in MC recruitment in vivo have never been investigated.

The aim of this study was to identify the role of Hh proteins in SMC recruitment by neo-vessels and to characterize their mechanism of action. As such, we conducted a comprehensive series of experiments to demonstrate that Shh contributes to PDGF-BB-induced SMC migration in vitro and drives mural cell recruitment on growing vessels in the mouse corneal angiogenesis model.

\section{Materials and methods}

\section{Products}

Recombinant Shh, PDGF-BB and Vascular Endothelial Growth Factor (VEGF) were purchased from R\&D Systems, Smoothened (Smo) inhibitor cyclopamine from Sigma and SANT2 from Enzo Life Sciences. PI3K inhibitors AS-252424 and Wortmannin, and PDGFR- $\beta$ inhibitor Imatinib were purchased from Santa Cruz.

\section{Cell culture and migration assays}

Rat aortic SMCs were obtained from Lonza. They are maintained in DMEM:F12 (1:1) medium supplemented with $20 \%$ fetal bovine serum, in humidified $5 \% \mathrm{CO}_{2}$ atmosphere at $37^{\circ} \mathrm{C}$ and used between passages 2 to 12 . Quiescent SMCs were obtained by a 24 hour culture in serum free medium. Commercial (Lonza) rat aortic SMC phenotype was characterized by immunocytochemistry with anti- $\alpha$-smooth muscle actin ( $\alpha$-SMA) (Sigma), anti-nerve/glial antigen-2 (NG2) (Millipore) and anti-PDGFR- $\beta$ (R\&D Systems) antibodies (Fig S1A). They demonstrated same phenotypic properties than aortic SMCs in situ (Fig S1B). 
For Transwell chemotactic assay, BD Falcon ${ }^{\mathrm{TM}}$ inserts were seeded with 65000 cells/well. Compounds to be tested were added in lower compartment. Migration was assessed as the mean of cells present below the porous membrane in 9 fields per well 6 hours after seeding.

Wound-healing assay was performed in 12 wells culture plate. A confluent layer of SMCs was scratched by a plastic tip and pictures were shot at beginning and 72 hours later.

Random motility test was performed on glass slides. SMC were tracked using a time lapse Zeiss microscope. More than 60 cells per condition were individually tracked by shooting pictures each 15 min for 15 hours (Axio Vision software). Each condition of the migration and motility assays was assayed in triplicate, and each experiment performed at least three times.

When indicated culture medium was supplemented with PDGF-BB $(10 \mu \mathrm{g} / \mathrm{mL})$, recombinant Shh (1 $\mu \mathrm{g} / \mathrm{mL}$ ), anti-Hh blocking antibody 5E1 (Developmental Studies Hybridoma Bank) (1.5 $\mu \mathrm{g} / \mathrm{mL}$ ), cyclopamine ( $1 \mu \mathrm{mol} / \mathrm{L})$ GANT58 (Tocris Biosciences) $(5 \mu \mathrm{mol} / \mathrm{L}),(\mathrm{GANT} 61$ (Alexis) $(5 \mu \mathrm{mol} / \mathrm{L})$ or PI3K $\gamma$ selective inhibitor (AS252424) (100 nmol/L).

siRNA transfection and adenovirus transduction

Cells were transfected with siRNA (Eurogentec) using Interferin (Polyplus transfection ${ }^{\mathrm{TM}}$ ) according to the manufacturer's instructions. Migration assays were performed 48 hours after transfection of quiescent cells. Adenovirus encoding for an inactive form of PI3K $\gamma$ (KRPI3K $\gamma$ ) and control adenovirus were obtained from M Laffargue's lab ${ }^{25}$. Cells were transduced at 50 MOI overnight then washed twice with serum free medium. Fresh medium was added for 24 hours before using cells.

\section{RNA extraction, reverse transcription, polymerase chain reaction and real-time quantitative PCR}

Total RNA was extracted from cultured SMCs or from mouse corneas, according to the TriReagent manufacturer's instructions (Molecular Research Center Inc.). Quantitative RT-PCR was performed as detailed in supplemental data using SYBR green technique. Primer sequences are described in supplemental data.

\section{Western blot and immunostaining techniques}

Antibodies used in western blot analysis were: anti-Akt, anti-phospho-Akt (Ser473), anti-ERK1/2 and anti-phospho-ERK1/2 (Tyr202/Tyr204) (Cell Signaling Technology). Primary antibodies were then incubated with secondary Alexa-700 nm and Alexa-750 nm coupled antibodies (Invitrogen) and Alexa-800 $\mathrm{nm}$ coupled antibodies (LI-COR) and resolved with an infrared system (OdysseyR, LI-COR).

Concentration of Shh protein in cultured SMC media was measured using the ELISA Kit "Mouse Sonic Hedgehog N-Terminus Quantikine" (R\&D Systems). Hh immunodetection on cells was performed with mouse anti-Hh antibody 5E1 (Developmental Studies Hybridoma Bank). Detection of PDGF-BB, Shh, ECs and $\mathrm{NG}^{+} \mathrm{MC}$ in the cornea model was performed with antiPDGF-B subunit antibody (Santa Cruz.), anti-C terminal Shh antibody (R\&D systems), antiCD31 antibody (BMA) and anti-NG2 antibody (Millipore) and revealed with fluorescent dye (Alexa 488, 564 and 647) coupled secondary antibodies (Invitrogen). Slides were mounted with Vectashield (Vector) imaging medium. Images were captured at $22^{\circ} \mathrm{C}$ with Olympus FV1000 confocal microscope or Zeiss Observer Z1 microscope equipped with a Zeiss digital camera. 3D Image reconstruction was performed with Imaris Bitmap software.

\section{Mouse cornea pocket angiogenesis assay}

$\mathrm{C} 3 \mathrm{HeB} / \mathrm{FeJ}$ mice were obtained from Charles River Laboratories and bred in our animal facility. Mice were handled in accordance with the guidelines established by the National Institute of Health and Medical Research (INSERM) and approved by the local Institutional Animal Care and 
Use Committee $\mathrm{N}^{\circ}$ A501303-A. VEGF pellets were prepared and implanted in the corneas of 45-week-old $\mathrm{C} 3 \mathrm{HeB} / \mathrm{FeJ}$ mice as previously described ${ }^{26}$. Pellets contain $5 \mu \mathrm{g}$ VEGF and $20 \mathrm{nmol}$ of Cyclopamine or of Imatinib as indicated. Pellets without growth factor were implanted in control mice. Vessel formation and stability were examined by immunostaining 5 or 28 days after implantation.

\section{Statistics}

Results are reported as mean \pm SD. Comparisons within groups were performed using ANOVA. Comparisons between individual groups were performed with the Mann-Whitney test.

\section{$\underline{\text { Results }}$}

\section{Hh proteins and Hh pathway activation are required for PDGF-BB-induced SMC migration}

During angiogenesis, ECs have been shown to produce factors necessary for MC recruitment including PDGF-BB ${ }^{27}$.

Recent data demonstrated that Shh contributes to PDGF-BB-stimulated fibroblast migration ${ }^{20}$. As PDGF-BB is one of the main chemotactic factors for SMCs, we hypothesized that Hh proteins could work in combination with PDGF-BB to promote SMC migration. As shown in Fig. 1A, Hh blocking antibody 5E1 decreased PDGF-BB-induced migration of commercial rat aortic primarycultured SMCs . To confirm this data and further investigate whether activation of $\mathrm{Hh}$ signaling is necessary for PDGF-BB-induced SMC migration, we inhibited Smo activity with cyclopamine and SANT2. The two inhibitors decreased PDGF-BB chemotactic activity (Fig. 1A and data not shown for SANT2). Taken together, these data demonstrate that Hh proteins and their subsequent activation of $\mathrm{Hh}$ signaling participate to PDGF-BB-induced SMC migration.

As cultured vascular SMCs express the main $\mathrm{Hh}$ signaling pathway elements including $\mathrm{Hh}$ receptor Patched-1 (Ptch-1), Smoothened (Smo), and Glioma-associated oncogene 1 (Gli1) transcription factor (Fig. S2), we investigated the chemotactic effect of Hh proteins on SMCs. In contrast to PDGF-BB, Shh did not induce aortic SMC chemotaxis even at a wide range of concentrations (10 ng/mL to $1 \mu \mathrm{g} / \mathrm{mL}$ ) (Fig. 1B) or long lasting migration time (24 hours) (Fig. S3). Similarly, $1 \mu \mathrm{g} / \mathrm{mL}$ of Ihh or Dhh did not show any chemotactic effect on SMCs (Fig. 1B). These data demonstrate that despite their involvement in PDGF-BB driven SMC migration, Hh proteins alone are not chemotactic for SMCs.

We then tested whether Shh was able to promote SMC chemokinesis in the wound-healing assay, and in the random motility test. We found that Shh was able to stimulate SMC motility in these two tests independently of the presence of a concentration gradient (Fig 1C).

Altogether these data suggest that Shh promotes PDGF-BB-induced SMC migration by stimulating SMC motility without inducing chemotaxis.

\section{Shh expression is stimulated by PDGF-BB}

To determine whether PDGF-BB-treated SMCs were capable of producing Hh proteins, we measured mRNA level of each Hh in SMCs by RT-qPCR. In the absence of PDGF-BB, Shh was the main Hh expressed $\left(1.3 \times 10^{4} \mathrm{mRNA}\right.$ copy/ $\mu \mathrm{g}$ RNA); Ihh was faintly expressed while Dhh mRNA was in the range of the detection threshold (Fig. 2A). Moreover, when SMCs were stimulated by PDGF-BB for 6 hours, Shh mRNA level increased by 3.2 fold as compared to control unstimulated quiescent SMCs, while Ihh and Dhh expression remained unchanged (Fig. 2A). The increase in Shh mRNA level became significant after a 4 hour PDGF-BB-stimulation (Fig. 2B). Shh protein accumulated in the cytoplasm and at the plasma membrane of PDGF-BBstimulated SMCs (Fig. S4), and was secreted by PDGF-BB-stimulated SMCs more abundantly 
than by control unstimulated SMCs (secreted Shh was 50 times more concentrated) (Fig. 2C) in a dose dependent manner (Fig. 2D).

Additionally, when Shh expression was specifically inhibited by Shh-siRNA, PDGF-BB-induced SMC migration was decreased by at least $50 \%$ (Fig. 1A and S5). Together these data demonstrate that Shh expression is induced by PDGF-BB and identified Shh as the Hh protein mediating PDGF-BB-induced SMC migration (see condensed data in Fig. 7).

Since Gli1 overexpression is one of the main reporters of $\mathrm{Hh}$ signaling pathway activation, we first verified whether Gli1 expression increases upon PDGF-BB stimulation (Fig. S6A). The role of Shh in PDGF-BB-induced Gli1 overexpression was controlled by Hh blocking antibodies (5E1). Moreover, we confirmed the involvement of Gli1 in PDGF-BB-induced SMC migration by using 3 Gli1 inhibitors including 2 small molecules: GANT58 and GANT61 (Fig. S6B) and the physiological inhibitor of Gli1: the N-terminal part of Gli3 (Fig. S6C and S6D). All 3 inhibitors significantly reduced PDGF-BB-mediated SMC migration. All together these data confirm the activation of Hh canonical signaling pathway by PDGF-BB.

\section{Shh-dependent activation of PI3K $\gamma$ and ERK1/2 participates in PDGF-BB-induced SMC migration}

Two of the main intracellular signaling pathways activated by PDGF-BB are MAPK and PI3K signaling, which lead to ERK1/2 and Akt phosphorylation, respectively.

Previous studies have shown that Shh activates PI3K and subsequently leads to Akt phosphorylation, in various cell types ${ }^{21,23,28-30}$. We demonstrated that recombinant Shh is capable of inducing a fast (15 min) Akt phosphorylation in SMCs. We showed that Shh-induced Akt phosphorylation is entirely inhibited by the pan PI3K inhibitor Wortmannin, the PI3K $\gamma$ selective inhibitor AS-252424 (Fig. 3A) and by competition with the inactive mutant of PI3K ${ }^{25}$ (Fig. S7A), demonstrating that Shh-induced Akt phosphorylation is mediated via PI3K $\gamma$. In contrast, the fast Akt phosphorylation induced by PDGF-BB was only inhibited by Wortmannin (Fig. 3A) but not by AS-252424 or the competitive inactive form of PI3K $\gamma$ (Fig. S7A), confirming that PDGF-BB-induced Akt phosphorylation is rather mediated by $\mathrm{PI} 3 \mathrm{~K} \alpha / \beta / \delta$.

After PDGF-BB stimulation of SMCs, Akt phosphorylation demonstrated a rapid and transient increase (maximal at $15 \mathrm{~min}$ ), then a rapid decrease, followed by a second wave of activation (Fig. 3B). In the presence of the Hh blocking antibody 5E1, the delayed second wave of Akt phosphorylation was strongly decreased suggesting that the late activation of Akt was dependent on Shh autocrine action. Indeed, the early PDGF-BB-induced Akt phosphorylation was totally inhibited by Wortmannin (Fig. 3A), but not by the PI3K $\gamma$ specific inhibitor (Fig. 3C). In contrast, the delayed Akt phosphorylation was strongly decreased by the PI3K $\gamma$ inhibitor (Fig. 3C), suggesting that this delayed activation is due to Shh-induced PI3K $\gamma$ activation.

Finally, we demonstrated that PI3K $\gamma$ inhibition by a selective drug (AS-252424) or in the inactive PI3K $\gamma$ mutant-transduced cells, reduced PDGF-BB-induced SMC migration by more than 50\% (Fig. 3D). We verified that PI3K $\gamma$ inhibitors block Shh-induced motility (Fig. 3E and S7B). Together these data suggest that Shh-activated PI3K $\gamma$ is one major determinant of PDGF-BBinduced SMC migration.

We also showed that recombinant Shh activates MAPK pathway leading to ERK1/2 phosphorylation (Fig. 4A). Kinetic analysis demonstrated a delayed reactivation of ERK1/2 phosphorylation after a 4 hour-PDGF-BB stimulation (Fig. 4B and 4C). In the presence of the Hh blocking antibody 5E1 or the Smo inhibitor cyclopamine, the early wave of ERK1/2 activation was not affected while the delayed activation was strongly disrupted (Fig. 4B, 4C, 4D and 4E), suggesting that PDGF-BB-induced Shh mediates the late ERK1/2 phosphorylation through Hh signaling pathway activation and may also participate, via this pathway, in SMC migration. 


\section{Shh is required for PDGF-BB-mediated MC recruitment on neo-vessels in the mouse cornea}

We investigated Shh involvement in MC recruitment during neo-vessel formation in vivo using a mouse cornea micro-pocket angiogenesis assay.

VEGF pellet implantation strongly increased Shh expression (Fig. 5A) mainly at the leading edge of neovessels (Fig. 5B and 5C). At the tip of the growing vessel we found both CD31+ ECs and a SMC subpopulation characterized by the expression of $\alpha$-SMA, NG2 proteoglycan marker and PDGFR- $\beta$ (Fig. S7). Shh expression was mainly colocalized with the NG2+ expressing cells at the interface between ECs and SMCs (Fig. 5D).

To test whether Shh expression was induced by PDGF-BB in vivo, we first verified that Pdgfb was increased after VEGF pellet implantation (Fig. S8). Then imatinib, an inhibitor of PDGFR phosphorylation was added together with VEGF in the pellet. In this condition VEGF-induced Shh mRNA expression in neovessels was strongly reduced, demonstrating that PDGF-BB is required for induction of Shh expression by VEGF in vivo (Fig. 5A).

The role of Shh in MC recruitment was investigated using the Hh pathway inhibitor cyclopamine. Five days after corneal implantation of pellets containing both VEGF and cyclopamine, the growing new vessels showed significant decrease in the number of recruited NG2+ MC (Fig. 6A and $6 \mathbf{F}$ ). The number of vessels, their diameters and vessel network connections remained unchanged (Fig. 6C, 6D and 6E). An identical decrease in the number of recruited $\mathrm{NG}^{+} \mathrm{MC}$ was observed when PDGFR was inhibited with imatinib (Fig. 6A).

Twenty eight days later, as expected inhibition of PDGF pathway by imatinib led to a vessel destabilization and a complete blood vessels regression. Cyclopamine-treated corneas were less drastically affected but also showed a marked reduction in blood vessel number (Fig. 6B and 6C), diameter and network connections (Fig. 6D and 6E), suggesting that Shh pathway is involved in maintaining/improving neovessel lifespan (Fig. 6F). Altogether, those latter data demonstrate the role of Shh expression in neo-vessel maturation and stabilization in vivo. Moreover, it confirms that Shh acts downstream of PDGF-BB.

NG2+ MC early recruitment in the leading edge of growing vessel does not require their proliferation

Since Shh was previously shown to promote SMC proliferation in vitro ${ }^{16-18}$ and to drive the proliferative effect of PDGF-BB ${ }^{20}$, we checked the role of $\mathrm{Hh}$ pathway in $\mathrm{NG2+} \mathrm{MC}$ proliferation in vivo.

NG2+ MC proliferation was examined after mice were administered with BrdU, 3 and 5 days after induction of angiogenesis by VEGFA. Double BrdU+ NG2+ labeled cells were found on the preexisting parental/limbal vessels (Fig. S9B) while we did not find any proliferating NG2+ cells in the growing new blood vessels either at days 3 or 5 (Fig. S9A and S9C) demonstrating that $\mathrm{NG} 2+$ cell recruitment at the tip of the growing vessels does not require cell proliferation at least during the first 5 days.

Thus these data suggest that new vessel formation in the mouse cornea mainly involves vessel remodeling with endothelial and mural cell migration at least during the first days after angiogenesis was induced, and that these processes are dependent on the Shh pathway.

Moreover, we found that addition of cyclopamine did not reduce the number of proliferating NG2+ cells on limbal vessels (Fig. S9D) demonstrating that activation of the Hh signaling pathway is not necessary for MC proliferation in this model of angiogenesis.

\section{$\underline{\text { Discussion }}$}

Previous investigations have shown that the morphogen Shh is upregulated in different pathologic conditions such as ischemia ${ }^{12}$, and enhances revascularization of ischemic tissues. In the 
ischemic heart, exogenous Shh administration not only promotes neovascularization, but also increases vessel muscularization, indicating that Shh may play a role in vessel maturation ${ }^{14}$. In the present study, we confirmed the essential role of Shh in blood vessel muscularization and revealed that Shh participates in PDGF-BB-induced SMC migration by increasing cell motility but not chemotaxis while PDGF-BB drives the direction of the movement. Indeed, we next demonstrated that both Shh expression and secretion were increased after SMC stimulation by PDGF-BB and that autocrine production of Shh was involved in PDGF-BB-stimulated SMC migration. This finding reveals a new effect of Shh on SMCs, in addition to its previously demonstrated role in proliferation of these cells ${ }^{16-18}$.

Even if Hh proteins are suggested to act on angiogenesis only indirectly ${ }^{12,31,32}$, ECs were shown to respond to Shh by increasing their migration and the impermeability of the brain endothelium ${ }^{33}$. In the context of growing vessels, SMC-derived Shh may not only act on SMCs themselves, but also on the neighborhood ECs by stimulating their migration and consequently by promoting vessel growth. However, our data showed that inhibition of the Hh signaling pathway has no effect on vessel growth at least during the first days after induction suggesting that Hh has only minor effect on this process. Moreover, an effect of Shh on endothelial permeability of new formed corneal vessel cannot be rule out, but the fact that Shh is mainly produced at the edge of growing vessels suggests that this effect remains probably minor.

Moreover, this study characterizes Shh-dependent signaling in SMCs and demonstrates that PDGF-BB-stimulated Shh induces the canonical Hh signaling pathway and a delayed activation of ERK1/2 and PI3K/Akt phosphorylation, two key mediators of the cell migration process. Indeed, PI3K targets $\left(\operatorname{PtdIns}(4,5) \mathrm{P}_{2}\right.$ and $\left.\operatorname{PtdIns}(3,4,5) \mathrm{P}_{3}\right)$ mediates cell migration via several downstream molecules, such as protein kinase $\mathrm{B}$ (PKB/Akt), guanine nucleotide exchange factors (GEFs), GTPase activating proteins, (GAPs) and protein tyrosine kinases that control cell movement ${ }^{34}$. To sustain this process, PI3K requires to be reactivated by a Rho GTPasedependent activation loop ${ }^{34}$. The potentiating effect of Shh appears to be mediated by Smo, a member of the GPCR superfamily ${ }^{35}$. In contrast to PDGFR- $\beta$, a tyrosine kinase receptor, which activates PI3K $\alpha, \beta$ and $\delta$ isoforms, GPCRs are known to activate the PI3K $\gamma$ subclass ${ }^{36}$. Our data demonstrate, for the first time, that Shh activates PI3K $\gamma$ via Smo, and that PDGF-BB-mediated $\mathrm{Shh} / \mathrm{Smo}$ signaling leads to a delayed $\mathrm{PI} 3 \mathrm{~K} \gamma$ activation, allowing long lasting cell activation which finally increases PDGF-BB-induced migration.

Previous data demonstrated that $\mathrm{PI} 3 \mathrm{~K} \gamma$ was required for reparative angiogenesis by contributing to angiogenic factor production by ECs ${ }^{37}$. We present here strong evidence that Shh-activated $\mathrm{PI} 3 \mathrm{~K} \gamma$ is one of the events triggering PDGF-BB-induced SMC chemotaxis and could potentially also be involved in MC recruitment. Cotreatment with both $\mathrm{Hh}$ and PI3K $\gamma$ inhibitors had a stronger inhibitory effect than $\mathrm{Hh}$ inhibitors alone suggesting that $\mathrm{PI} 3 \mathrm{~K} \gamma$ activity is not exclusively regulated by Shh (Fig. S10). Interestingly, autocrine production of Monocyte Chemotactic Protein-1 (MCP-1), another GPCR agonist, has also been shown to implicate PI3K $\gamma$ in PDGF-BB-induced aortic SMC migration ${ }^{25}$. Our study brings to light a novel function of Shh, implicating this morphogenic protein in PDGF-BB-induced SMC chemotaxis in a similar fashion to osteopontin ${ }^{38}$ or MCP-1 ${ }^{25}$. Shh is expressed and secreted under PDGF-BB stimulation of SMCs and triggers an array of intracellular pathways (PI3K $\gamma$ and ERK1/2) which converge to allow long term PDGF-BB-induced signaling mechanisms to initiate the cellular response, ie SMC migration.

$\mathrm{MC}$ coverage of growing vessels is the result of different processes including MC proliferation, and migration from preexisting vessels or MC differentiation from progenitor cells. MC include smooth muscle lineage cells at different differentiation stage such as pericytes and SMC subtypes ${ }^{39}$. In mouse cornea, we found that MC of growing vessels co-express $\alpha$-SMA, NG2 and PDGFR$\beta$ that are commonly associated to phenotypes of human pericytes (Fig. S11A, S11B and S12A) and rat aortic SMCs (Fig. S1). Moreover, the migratory response to PDGF-BB of these mural 
cells from human and rat depends identically on the Hh pathway (Fig. S12B). These findings suggest that data from in vitro experiments of rat aortic SMCs are relevant to understand mechanisms of PDGF-dependent $\mathrm{MC}$ recruitment in vivo at least in the mouse corneal angiogenesis model.

In the pathophysiological context, notably during angiogenesis, PDGF-BB is one of the major factors involved in MC recruitment ${ }^{40}$. This growth factor is produced in neo-vessels, and is highly responsive to angiogenic factors, including VEGFA ${ }^{41,42}$. Expression of PDGF-BB by neovessels is essential for proper recruitment and organization of MCs, which express PDGFR- $\beta$ and can proliferate and migrate along the PDGF-BB gradient generated by ECs ${ }^{43}$. Our in vivo data show that Shh is required for the previously characterized effects of PDGF-BB on vessel maturation. Blocking either PDGF-BB or Shh action, strongly reduced $\mathrm{NG}^{+}{ }^{+} \mathrm{MC}$ recruitment and consequently reduced both vessel maturation and life span.

Because blocking Hh signaling pathway was well shown to inhibit SMC proliferation in vitro ${ }^{16-18}$ the effect of Shh on MC recruitment in vivo may be the result of its effect on MC proliferation instead of migration. However, our data show that in the cornea, early steps of new vessel formation do not involve either EC or MC proliferation and moreover that blocking Hh signaling pathway does not reduce cell proliferation on preexisting limbal vessels thus suggesting that inhibition of MC proliferation is probably not the target of Hh inhibitors. Our in vitro data, demonstrating that Shh is involved in PDGF-BB-mediated SMC migration, suggest that one potential effect of Shh in vivo, is to promote this effect.

In summary, the present study demonstrates that i) Shh expression is induced by PDGF-BB in MCs both in vivo in the mouse corneal angiogenesis model and in vitro in cultured SMCs; ii) inhibition of Shh signaling leads to a decrease in the MC number on new vessels and a subsequent decrease in vessel life span. Taken together, these findings strongly suggest that autocrine production of Shh by MCs is a downstream effector of PDGF-BB for MC migration and recruitment in neo-vessels (Fig. 8), documenting an enigmatic role of Shh in vessel maturation ${ }^{14}$. Finally, our work underscores the potential therapeutic application of Shh to increase the endogenous effect of PDGF-BB in promoting vessel maturation by enhancing MC migration towards PDGF-BB-producing ECs.

\section{Acknowledgments}

For their excellent technical assistance, we thank Jérôme Guignard (INSERM U1034, Pessac) at the animal facility, Annabel Reynaud for molecular biology experiments and Myriam Petit for histology.

\section{Sources of Funding}

This study was supported by grants from the Conseil Régional d'Aquitaine (action inter-régionale Aquitaine-Midi Pyrénées); Communauté de Travail des Pyrénées; ANR, program (ANR-07PHYSIO-010-02); Fondation Recherche Médicale, program on cardiovascular aging (DCV20070409258); post doctoral fellowship from La Ligue Nationale Contre le Cancer (MA $\mathrm{R})$.

\section{Authorship contribution}

Yao Qinyu performed research, analyzed data and wrote paper, Renault Marie-Ange performed research, analyzed data and edited the paper, Chapouly Candice performed research, Vandierdonck Soizic performed research, Belloc Isabelle performed research, Jaspard-Vinassa Beatrice performed research, Daniel-Lamazière Jean-Marie analyzed data, Laffargue Muriel contributed vital new reagents, Merched Aksam designed research and edited the paper, 
Desgranges Claude designed research, performed research, analyzed data and wrote the paper and Gadeau Alain-Pierre designed research, wrote the paper.

\section{$\underline{\text { Disclosures }}$}

None

\section{$\underline{\text { References }}$}

1. Carmeliet P. Angiogenesis in health and disease. Nat Med. 2003;9(6):653-660.

2. Ribatti D, Nico B, Crivellato E. The role of pericytes in angiogenesis. Int J Dev Biol. 2011;55(3):261-268.

3. Gerhardt H, Betsholtz C. Endothelial-pericyte interactions in angiogenesis. Cell Tissue Res. 2003;314(1):15-23.

4. Hellberg C, Ostman A, Heldin CH. PDGF and vessel maturation. Recent Results Cancer Res. 2010;180:103-114.

5. Hellstrom M, Kalen M, Lindahl P, Abramsson A, Betsholtz C. Role of PDGF-B and PDGFR-beta in recruitment of vascular smooth muscle cells and pericytes during embryonic blood vessel formation in the mouse. Development. 1999;126(14):3047-3055.

6. Hirschi KK, Rohovsky SA, Beck LH, Smith SR, D'Amore PA. Endothelial cells modulate the proliferation of mural cell precursors via platelet-derived growth factor-BB and heterotypic cell contact. Circ Res. 1999;84(3):298-305.

7. Nystrom HC, Lindblom P, Wickman A, et al. Platelet-derived growth factor B retention is essential for development of normal structure and function of conduit vessels and capillaries. Cardiovasc Res. 2006;71(3):557-565.

8. Arkonac BM, Foster LC, Sibinga NE, et al. Vascular endothelial growth factor induces heparin-binding epidermal growth factor-like growth factor in vascular endothelial cells. J Biol Chem. 1998;273(8):4400-4405.

9. Schmidt NO, Koeder D, Messing M, et al. Vascular endothelial growth factorstimulated cerebral microvascular endothelial cells mediate the recruitment of neural stem cells to the neurovascular niche. Brain Res. 2009;1268:24-37.

10. Nilsson I, Shibuya M, Wennstrom S. Differential activation of vascular genes by hypoxia in primary endothelial cells. Exp Cell Res. 2004;299(2):476-485.

11. Nagase T, Nagase M, Machida M, Fujita T. Hedgehog signalling in vascular development. Angiogenesis. 2008;11(1):71-77.

12. Pola R, Ling LE, Silver M, et al. The morphogen Sonic hedgehog is an indirect angiogenic agent upregulating two families of angiogenic growth factors. Nat Med. 2001;7(6):706-711.

13. Straface G, Aprahamian T, Flex A, et al. Sonic hedgehog regulates angiogenesis and myogenesis during post-natal skeletal muscle regeneration. J Cell Mol Med. 2009;13(8B):2424-2435.

14. Kusano KF, Pola R, Murayama T, et al. Sonic hedgehog myocardial gene therapy: tissue repair through transient reconstitution of embryonic signaling. Nat Med. 2005;11(11):1197-1204.

15. Dohle E, Fuchs S, Kolbe M, Hofmann A, Schmidt H, Kirkpatrick CJ. Comparative study assessing effects of sonic hedgehog and VEGF in a human co-culture model for bone vascularisation strategies. Eur Cell Mater. 2011;21:144-156. 
16. Li F, Duman-Scheel M, Yang D, et al. Sonic hedgehog signaling induces vascular smooth muscle cell proliferation via induction of the G1 cyclin-retinoblastoma axis. Arterioscler Thromb Vasc Biol. 2010;30(9):1787-1794.

17. Morrow D, Cullen JP, Liu W, et al. Sonic Hedgehog induces Notch target gene expression in vascular smooth muscle cells via VEGF-A. Arterioscler Thromb Vasc Biol. 2009;29(7):1112-1118.

18. Wang G, Zhang Z, Xu Z, et al. Activation of the sonic hedgehog signaling controls human pulmonary arterial smooth muscle cell proliferation in response to hypoxia. Biochim Biophys Acta. 2010;1803(12):1359-1367.

19. Fabian SL, Penchev RR, St-Jacques B, et al. Hedgehog-Gli pathway activation during kidney fibrosis. Am J Pathol. 2012;180(4):1441-1453.

20. Yang L, Wang Y, Mao H, et al. Sonic hedgehog is an autocrine viability factor for myofibroblastic hepatic stellate cells. J Hepatol. 2008;48(1):98-106.

21. Polizio AH, Chinchilla P, Chen X, Kim S, Manning DR, Riobo NA. Heterotrimeric Gi proteins link Hedgehog signaling to activation of Rho small GTPases to promote fibroblast migration. J Biol Chem. 2011;286(22):19589-19596.

22. Renault MA, Roncalli J, Tongers J, et al. Sonic hedgehog induces angiogenesis via Rho kinase-dependent signaling in endothelial cells. J Mol Cell Cardiol. 2010;49(3):490-498.

23. Fu JR, Liu WL, Zhou JF, et al. Sonic hedgehog protein promotes bone marrowderived endothelial progenitor cell proliferation, migration and VEGF production via PI 3-kinase/Akt signaling pathways. Acta Pharmacol Sin. 2006;27(6):685-693.

24. Dunaeva M, Voo S, van Oosterhoud C, Waltenberger J. Sonic hedgehog is a potent chemoattractant for human monocytes: diabetes mellitus inhibits Sonic hedgehoginduced monocyte chemotaxis. Basic Res Cardiol. 2010;105(1):61-71.

25. Fougerat A, Smirnova N, Gayral S, et al. Determinant role of Phosphoinositide 3kinase gamma in MCP-1-mediated amplification of PDGF-induced aortic smooth muscle cell migration. Br J Pharmacol. 2012;166(5):1643-1653.

26. Kenyon BM, Voest EE, Chen CC, Flynn E, Folkman J, D'Amato RJ. A model of angiogenesis in the mouse cornea. Invest Ophthalmol Vis Sci. 1996;37(8):1625-1632.

27. Enge M, Bjarnegard M, Gerhardt H, et al. Endothelium-specific platelet-derived growth factor-B ablation mimics diabetic retinopathy. Embo J. 2002;21(16):4307-4316.

28. Elia D, Madhala D, Ardon E, Reshef R, Halevy O. Sonic hedgehog promotes proliferation and differentiation of adult muscle cells: Involvement of MAPK/ERK and PI3K/Akt pathways. Biochim Biophys Acta. 2007;1773(9):1438-1446.

29. Madhala-Levy D, Williams VC, Hughes SM, Reshef R, Halevy O. Cooperation between Shh and IGF-I in promoting myogenic proliferation and differentiation via the MAPK/ERK and PI3K/Akt pathways requires Smo activity. $J$ Cell Physiol. 2011;227(4):1455-1464.

30. Riobo NA, Lu K, Ai X, Haines GM, Emerson CP, Jr. Phosphoinositide 3-kinase and Akt are essential for Sonic Hedgehog signaling. Proc Natl Acad Sci U S A. 2006;103(12):4505-4510.

31. Renault MA, Chapouly C, Yao Q, et al. Desert hedgehog promotes ischemiainduced angiogenesis by ensuring peripheral nerve survival. Circ Res. 2013;112(5):762770 . 
32. Renault MA, Robbesyn F, Chapouly C, et al. Hedgehog-dependent regulation of angiogenesis and myogenesis is impaired in aged mice. Arterioscler Thromb Vasc Biol. 2013;33(12):2858-2866.

33. Alvarez JI, Dodelet-Devillers A, Kebir H, et al. The Hedgehog pathway promotes blood-brain barrier integrity and CNS immune quiescence. Science. 2011;334(6063):1727-1731.

34. Ward SG. Do phosphoinositide 3-kinases direct lymphocyte navigation? Trends Immunol. 2004;25(2):67-74.

35. Alcedo J, Ayzenzon M, Von Ohlen T, Noll M, Hooper JE. The Drosophila smoothened gene encodes a seven-pass membrane protein, a putative receptor for the hedgehog signal. Cell. 1996;86(2):221-232.

36. Stoyanov B, Volinia S, Hanck T, et al. Cloning and characterization of a G protein-activated human phosphoinositide-3 kinase. Science. 1995;269(5224):690-693.

37. Siragusa M, Katare R, Meloni M, et al. Involvement of phosphoinositide 3-kinase gamma in angiogenesis and healing of experimental myocardial infarction in mice. Circ Res. 2010;106(4):757-768.

38. Jalvy S, Renault MA, Leen LL, et al. Autocrine expression of osteopontin contributes to PDGF-mediated arterial smooth muscle cell migration. Cardiovasc Res. 2007;75(4):738-747.

39. von Tell D, Armulik A, Betsholtz C. Pericytes and vascular stability. Exp Cell Res. 2006;312(5):623-629.

40. Benjamin LE, Hemo I, Keshet E. A plasticity window for blood vessel remodelling is defined by pericyte coverage of the preformed endothelial network and is regulated by PDGF-B and VEGF. Development. 1998;125(9):1591-1598.

41. Kano MR, Morishita Y, Iwata C, et al. VEGF-A and FGF-2 synergistically promote neoangiogenesis through enhancement of endogenous PDGF-B-PDGFRbeta signaling. J Cell Sci. 2005;118(Pt 16):3759-3768.

42. Onimaru M, Yonemitsu Y, Fujii T, et al. VEGF-C regulates lymphangiogenesis and capillary stability by regulation of PDGF-B. Am J Physiol Heart Circ Physiol. 2009;297(5):H1685-1696.

43. Lindblom P, Gerhardt H, Liebner S, et al. Endothelial PDGF-B retention is required for proper investment of pericytes in the microvessel wall. Genes Dev. 2003;17(15):1835-1840.

\section{Legends}

Figure 1: Shh mediates PDGF-BB chemotactic effect on SMCs

(A). PDGF-BB (10 ng/mL)-stimulated and non-stimulated (Ctrl) SMC migration was assessed in the presence of the Smo-inhibitor, cyclopamine $(1 \mu \mathrm{mol} / \mathrm{L})$; the Hh blocking antibody, 5E1 (1.5 $\mu \mathrm{g} / \mathrm{mL}$ ); a siRNA directed against Shh ARN (siShh) (30 nmol/L); or their respective controls (IgG1, ethanol $0.005 \%(\mathrm{EtOH})$ and siCtrl). (B). SMC migration in response to various concentrations of Shh $(1 \mathrm{ng} / \mathrm{mL}$ to $1000 \mathrm{ng} / \mathrm{mL})$, Ihh $(1000 \mathrm{ng} / \mathrm{mL})$, Dhh $(1000 \mathrm{ng} / \mathrm{mL})$ and PDGF-BB (10 ng/mL). (C). SMC motility was tested for 72 hours in wound-healing assays and 15 hours in random motility assays with or without stimulation by recombinant Shh $(1 \mu \mathrm{g} / \mathrm{mL})$. Data represent means \pm SD of relative values versus Ctrl from 3 independent experiments performed in triplicate (NS: $p>0.05, * *: p<0.005$ and $* * *: p<0.0005$ ). 
Figure 2: PDGF-BB-stimulates Shh expression in SMCs

(A). SMCs were treated with $10 \mathrm{ng} / \mathrm{mL}$ PDGF-BB for 6 hours. mRNA copy number of Sonic (Shh), Desert (Dhh) and Indian (Ihh) Hh in stimulated (PDGF-BB) and unstimulated (Ctrl) SMCs was estimated by RT-qPCR. (B). Relative Shh mRNA expression in SMCs stimulated with PDGF-BB (10 ng/mL) for 2, 4 or 6 hours was determined by RT-qPCR; $\beta$-actin was used as housekeeping gene. $(\boldsymbol{C})$. Concentrations of secreted Shh protein present in 24 hour-conditioned culture media from PDGF-BB-stimulated SMCs or unstimulated (Ctrl) SMCs were quantified by ELISA. (D). Relative Shh mRNA expression in SMCs stimulated with different PDGF-BB concentrations was determined by RT-qPCR; Data represent means \pm SD from 3 independent experiments performed in triplicate (NS: non significant, *: $\mathrm{p}<0.05$ and $* * *: p<0.0005$ ).

Figure 3: PDGF-BB-induced SMC migration implicates Shh-mediated PI3K $\gamma$ activation (A). SMCs were pre-treated for 30 min with Wortmannin, a pan PI3K inhibitor (Wort), (100 $\mathrm{nmol} / \mathrm{L}$ ) or AS-252424, a PI3K $\gamma$ selective inhibitor (AS), (100 nmol/L), then stimulated for 15 min with PDGF-BB $(10 \mathrm{ng} / \mathrm{mL})$ or Shh $(1 \mu \mathrm{g} / \mathrm{mL})$. Proteins were extracted and processed by western blot before Akt and phosphorylated Akt (p-Akt) detection. (B) Kinetics of Akt phosphorylation in the presence or absence of the Hh blocking antibody $5 \mathrm{E} 1(1.5 \mu \mathrm{g} / \mathrm{mL}$ ) (Anti$\mathrm{Hh} \mathrm{ab}$ ), control IgG1, $(\boldsymbol{C})$ the $\mathrm{PI} 3 \mathrm{~K} \gamma$ selective inhibitor (PI3K $\gamma$ inhibitor) or DMSO, using western blot (left). Secondary Alexa-700 nm coupled antibodies (Invitrogen) and Alexa-800 nm coupled antibodies (LI-COR) were resolved with an infrared system (OdysseyR, LI-COR). Quantifications of band intensity performed with Odyssey software, are shown (right). Immunoblots shown are representative of 3 independent experiments. (D) Basal and PDGF-BBinduced SMC migration in presence or not of PI3K $\gamma$ inhibitor $(100 \mathrm{nmol} / \mathrm{L})(\mathrm{AS})$ or wortmannin $(100 \mathrm{nmol} / \mathrm{L})$ (Wort) or after transduction with empty (Ad-empty) or PI3K $\gamma$ inactive form (Ad$\mathrm{KR}$ ) adenoviruses. (E) SMC motility was measured after 72 hours in wound-healing assay and after 15 hours in random motility assay with or without stimulation by recombinant Shh (1 $\mu \mathrm{g} / \mathrm{mL})$ and in presence or absence of PI $3 \mathrm{~K} \gamma$ inhibitor $(100 \mathrm{nmol} / \mathrm{L})$. Data represent means $\pm \mathrm{SD}$ from 3 independent experiments performed in triplicate $(*: p<0.05$ and $* * *: p<0.0005)$.

Figure 4: Shh is required to sustain ERK1/2 phosphorylation induced by PDGF-BB

(A). Quiescent SMCs were stimulated for $15 \mathrm{~min}$ with Shh $(1 \mu \mathrm{g} / \mathrm{mL})$. Proteins were extracted and processed for detection of ERK (ERK1/2) and phosphorylated ERK1/2 (p-ERK1/2) by western blot. $(\boldsymbol{B})$. Kinetics of ERK1/2 phosphorylation in the presence or absence (Ctrl) of the Hh blocking antibody 5E1 $(1.5 \mu \mathrm{g} / \mathrm{mL})$ (anti-Hh ab). Immunoblots shown are representative of 3 independent experiments. $(\boldsymbol{C})$. Quantification of band intensity. (D). Kinetics of ERK1/2 phosphorylation in the presence or absence $(\mathrm{Ctrl})$ of the Smo inhibitor cyclopamine $(1 \mu \mathrm{mol} / \mathrm{L})$. Immunoblots shown are representative of 3 independent experiments. $(\boldsymbol{E})$. Quantifications of band intensity. $(*: \mathrm{p}<0.05)$.

\section{Figure 5: PDGF-BB-dependent Shh expression in VEGF-mediated corneal angiogenesis model}

VEGF $(5 \mu \mathrm{g})$ containing pellets were implanted into the cornea of $\mathrm{C} 3 \mathrm{HeB} / \mathrm{FeJ}$ mice $(\mathrm{n}=8$ per group). (A). Five days later, Shh mRNA level was evaluated by RT-qPCR in Ctrl and VEGFtreated corneas with or without PDGFR- $\beta$ inhibitor imatinib $(20 \mathrm{nmol}) ; \beta$-actin was used as housekeeping reference gene. Data of RT-qPCR represent means \pm SD of relative expression versus control corneas $(* *$ : $\mathrm{p}<0.005$; ***: $\mathrm{p}<0.0005)$. $(\boldsymbol{B})$. Shh (green, Alexa 488) and NG2 (Red, Alexa 568) expression were detected by immunostaining and imaged with a Zeiss Observer Z1 fluorescence microscope. ( $\boldsymbol{C}, \boldsymbol{D})$ Shh (green, Alexa 488), ECs (CD31, red, Alexa 568), NG2 (pink, Alexa 647) expression was revealed by immunohistology. Nuclei were counterstained 
using DAPI (blue). Images were shot with an Olympus FV1000 confocal microscope at low (x20) $(\boldsymbol{C})$ or high magnification $(\mathrm{x} 40)(\boldsymbol{D})$ respectively. In $(\boldsymbol{D})$ Right panel shows isosurface analysis by Imaris Bitmap software.

Figure 6: Inhibition of Hh signaling pathway stimulates neo-vessel maturation and stability VEGF $(5 \mu \mathrm{g})$ containing pellets in the presence or absence of Smo inhibitor (20 nmol cyclopamine, cyclop) or PDGFR- $\beta$ inhibitor ( $20 \mathrm{nmol}$ imatinib) were implanted into the cornea of $\mathrm{C} 3 \mathrm{HeB} / \mathrm{FeJ}$ mice ( $\mathrm{n}=8$ per group) for 5 days $(\boldsymbol{A})$ or 28 days $(\boldsymbol{B})$. ECs were immunostained using anti-CD31 antibody (red, Alexa 564)) and MCs with anti-NG2 ${ }^{+}$antibody (green, Alexa 488). Image capture was perform with Zeiss Observer Z1 microscope (x20)

Quantification of $(\boldsymbol{C})$ total vessel number, $(\boldsymbol{D})$ vessel diameter, $(\boldsymbol{E})$ vessel connections and $(\boldsymbol{F})$ number of $\mathrm{NG}^{+}$cells/vessel, 5 days or 28 days after VEGF pellet implantation in presence (gray histograms) or absence of cyclopamine (black). Data represent means \pm SD of relative values versus control corneas. NS: $p>0.05, * *: p<0.005 ; * * *: p<0.0005$.

Figure 7: Role of autocrine Shh signaling in the potentiation of PDGF-BB-induced SMC migration and recruitment

PDGF-BB secreted by VEGF-stimulated endothelial cells induces Shh expression in mural cells. Locally, autocrine Shh promotes a sequence of events mediated by downstream signaling pathways via PI3K $\gamma$ and ERK1/2, leading to co-stimulation of PDGF-BB pathway targets. By this novel mechanism, Shh potentiates PDGF-BB-mediated NG2+ mural cell migration and recruitment on growing vessels allowing their long-lasting stabilization. 
Figure 1

Figure 2

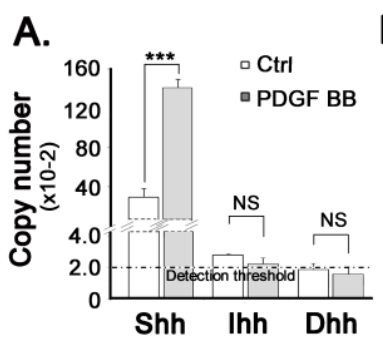

B.
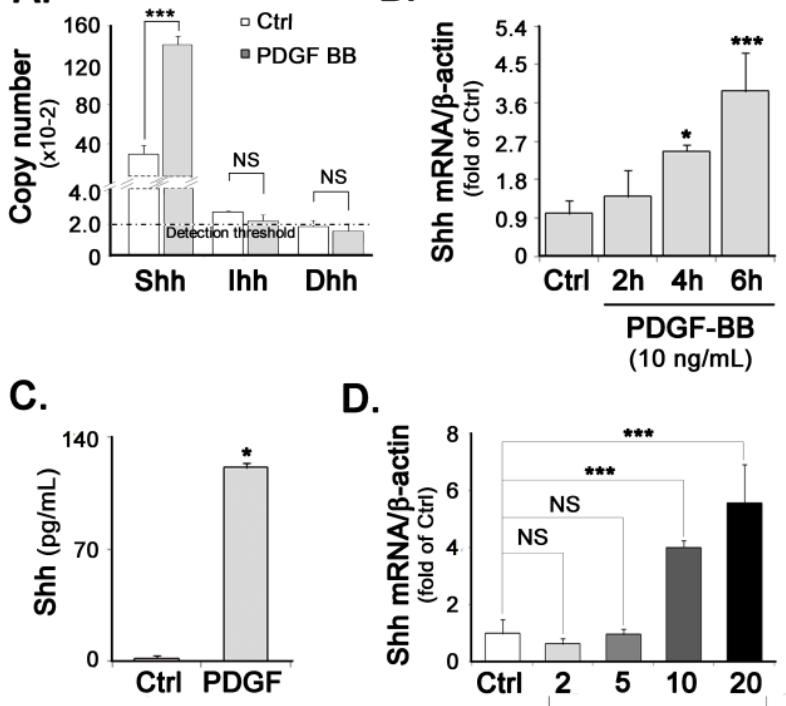

D.

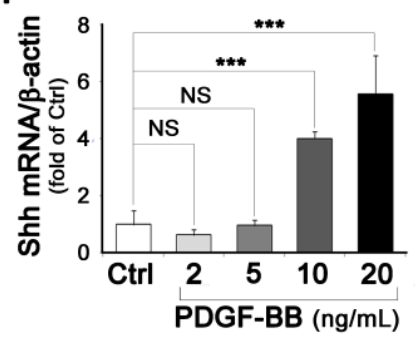


Figure 3

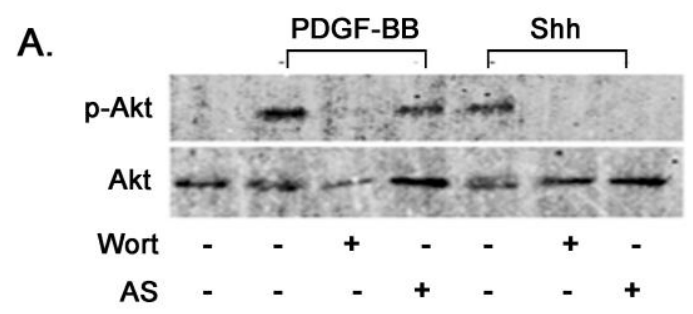

B.

PDGF-BB
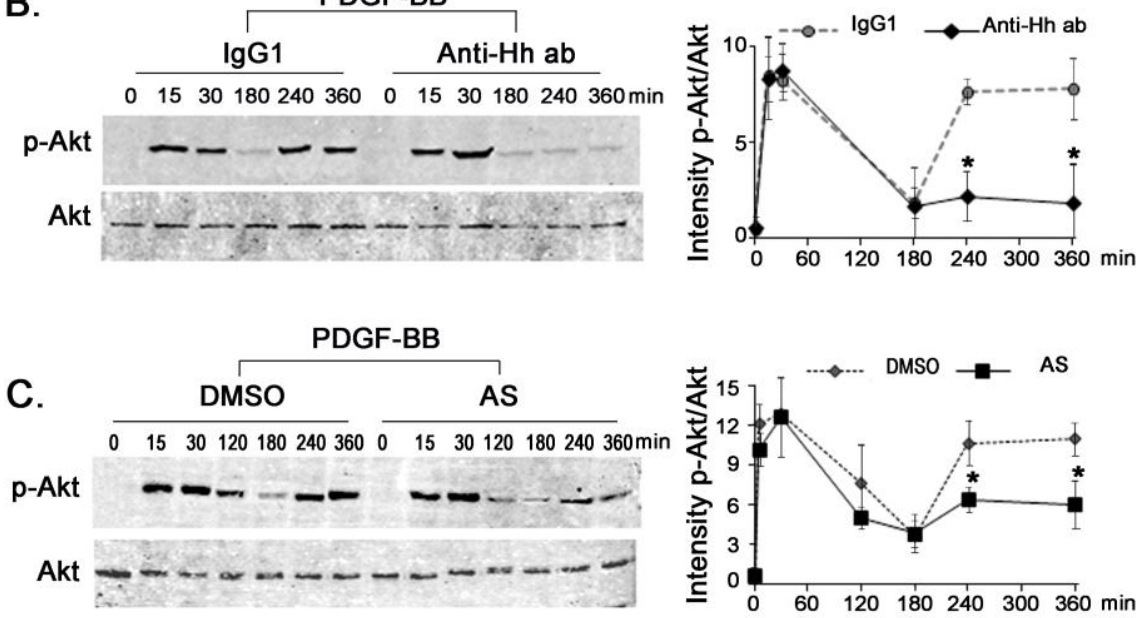

D.

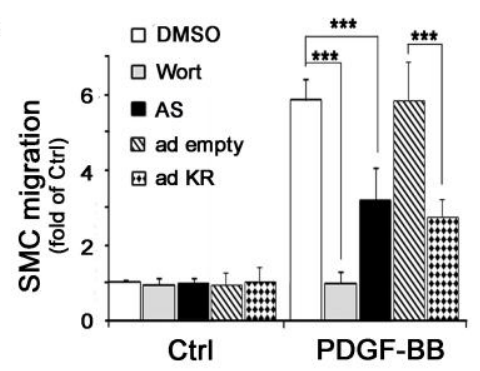

E.

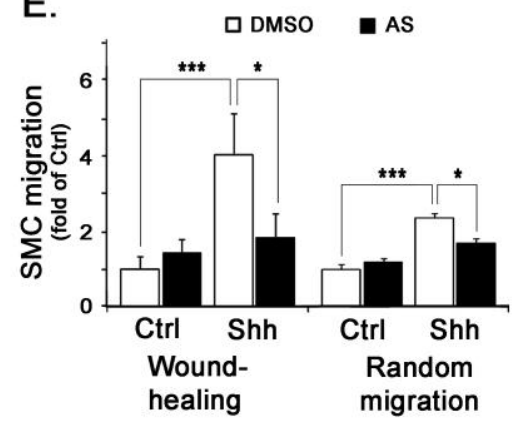


Figure 4

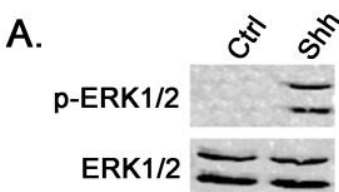

B.

PDGF-BB

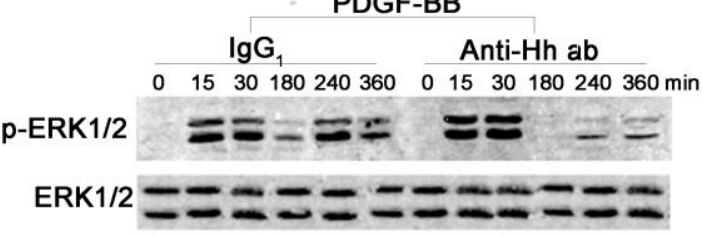

D.

PDGF-BB

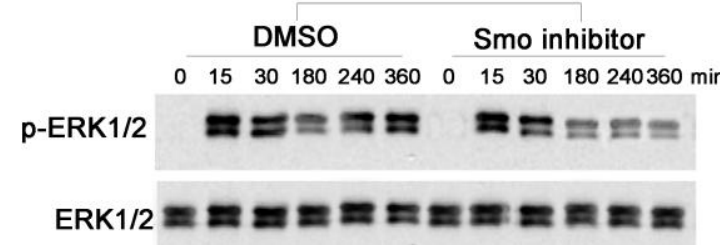

C.

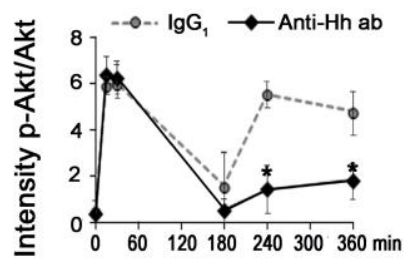

E.

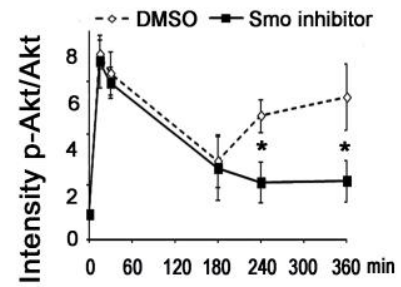


Figure 5

Figure 6
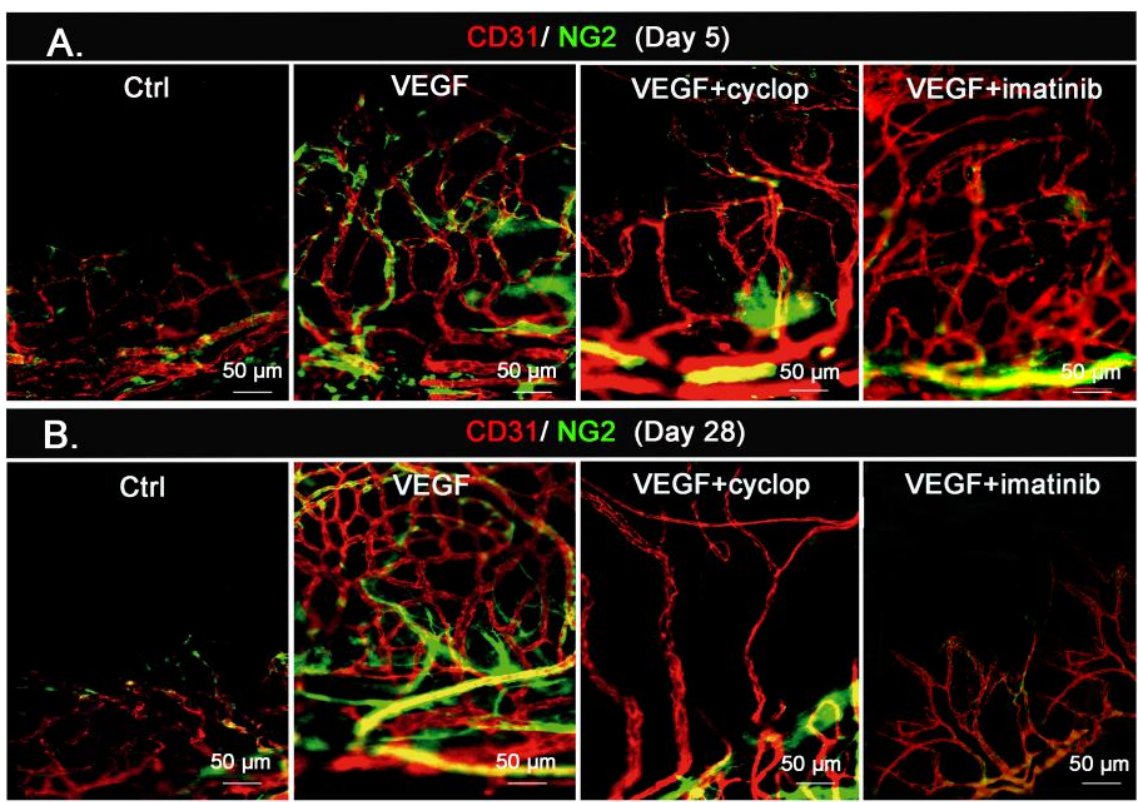

C.

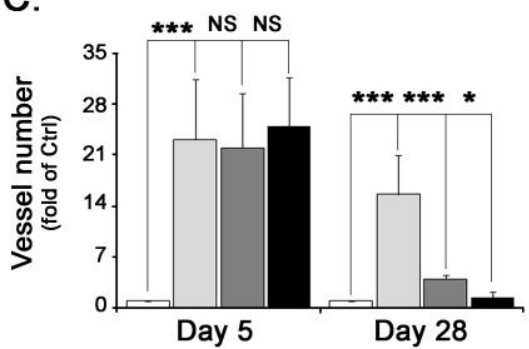

E.

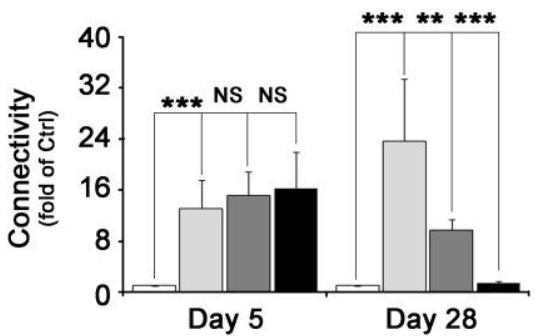

D.

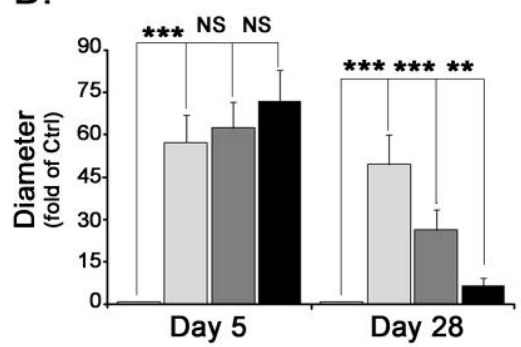

F.

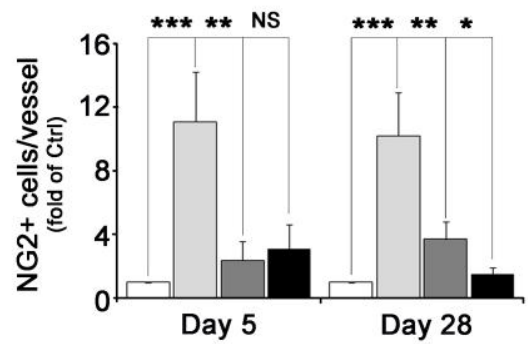

$\square$ Ctrl $\square$ VEGF $\square$ VEGF+cyclop $\square$ VEGF+imatinib 
Figure 7

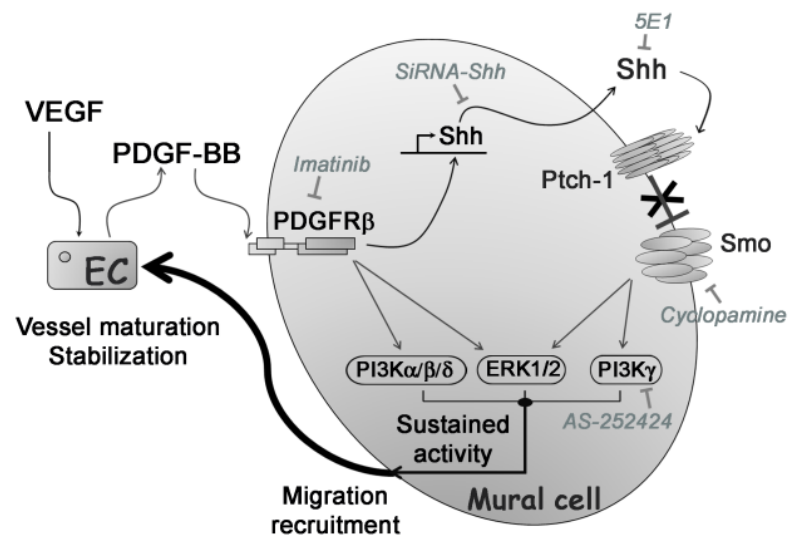

